\title{
General modeling of the windings for multi-phase ac machines
}

\section{Application for the analytical estimation of the mutual stator inductances for smooth air gap machines}

\author{
F. Scuiller ${ }^{1, a}$, E. Semail ${ }^{2,3}$, and J.-F. Charpentier ${ }^{1}$ \\ 1 Institut de Recherche de l'École Navale, 29240 Brest-Armées, France \\ 2 Arts et Métiers ParisTech, 59000 Lille, France \\ 3 Université Lille Nord de France, 59000 Lille, France
}

Received: 23 November 2009 / Accepted: 23 March 2010

Published online: 17 May 2010 - (c) EDP Sciences

\begin{abstract}
This paper, which deals with the winding modeling of ac multi-phase machines with a regular distribution of the stator slots, details an original matrix modeling of the stator winding. First, the properties of the balanced multi-phase windings (with integral-slot and fractional-slot patterns) are analysed. The winding function approach, one of the most common way to model the winding distribution effects on the stator rotating field, is then introduced. For multi-phase machines, it will be shown that the pole number generated by the winding distribution depends on a new parameter: the circularity index. The discrete nature of the winding, imposed by the stator slots, leads to the development of a discrete modeling of the winding obtained from sampling the winding function: two matrices, the winding function matrix and the distribution function matrix, are introduced to characterize the multi-phase winding. This matrix approach is thus a concise way to calculate the winding factors and to estimate the set of self and mutual stator inductances for smooth air gap multi-phase machines. A particularly original method of obtaining an analytical expression for the leakage mutual inductance is described. The results are validated with two experimental 5-phase PM machines by using experimental measurements and numerical simulations.
\end{abstract}

\section{Introduction}

Windings have been widely studied for three-phase machines: in the past, the main objective was to obtain sinusoidal magnetomotive forces [1,2]. This property implies good characteristics for machines supplied directly by three-phase grid voltages. With the advent of advanced current controls, the necessity of this winding constraint for the designer can be re-examined.

Studies on concentrated fractional windings [3-6] have been carried out especially on Permanent Magnet synchronous machines. For the manufacturers, these windings are attractive when compared with distributed windings because they allow shorter end-windings (with reduced losses [7,8] and easier winding processes [6]). However, they are also known for inducing parasitic effects $[9,10]$ particularly in the case of three-phase PM machines, because of the harmonics of the magnetomotive forces and electromotive forces. Consequently, this kind of windings is seldom used with three-phase machines that must provide high quality torque at low speeds: the reason is that high-performance vector controls require sinusoidal electromotive forces.

\footnotetext{
a e-mail: franck_scuiller@yahoo.fr
}

In the case of multi-phase machines with more than three phases [11-13], the constraints on the windings are not the same since multiple spatial and time harmonics can be tolerated when implementing vector controls that ensure high quality of torque [14-19]. Moreover, the harmonics yield an increase in the performances of the drive $[20,21]$ and a greater degree of fault tolerance in comparison with three-phase drives [22,23]. Multi-phase machines are thus attractive since a higher torque density and fault tolerance can be expected with a simpler design and with low torque pulsations $[19,24]$.

Nevertheless, the acceptance of more than one harmonic does not signify the acceptance of all the harmonics, especially for obtaining a torque with low torque ripples $[23,25,26]$. It is thus necessary to manage the levels of time and spatial harmonics. By using a Voltage Source Inverter controlled with a Pulse Width Modulation, it is nowadays possible to impose precise time harmonics on currents. By ensuring adequate design of the Permanent Magnet rotor and of the stator windings, it is possible to impose precise spatial harmonics.

The paper proposes a modeling of the windings using an approach which allows a rapid harmonic characterization of a large class of multi-phase windings so called balanced windings. Only rotating machines with a smooth air 
gap (no saliency effects) and with regular distribution of the slots along the stator periphery are considered. This modeling is used in the paper for the calculus of the inductances. Thanks to a matrix description that can be easily numerically implemented for a high number of phases, the modeling, considering the number of slots, takes into account the intrinsic discrete nature of a winding: this fact is important when harmonics must be taken into account as is the case for the determination of the inductances of the multiphase machines. Despite a first harmonic approach generally being sufficient to estimate the inductance of a three-phase machine, it is not at all the case for machines with more than three phases $[27,28]$.

Previous studies have already been dedicated to inductance calculus of multiphase machines with diametral concentrated windings: harmonics can then be easily taken into account $[20,29]$ in order to estimate synchronous inductances and windings factors. The same kind of approach can be applied with fractional-slot windings but the approach becomes too laborious if the designer wants to examine different pole/slot combinations in an optimization process. References concerning the winding modeling mainly relate to three-phase machines. The most common method is called Star of slots and is reviewed in $[3,30]$. This method is particularly well-adapted for the study of the fraction-slot configurations, despite not being originally designed for software implementation. Even though [3] provides elements to calculate the winding factors from the winding distribution in the slots (as in [6]), no relations are established to estimate the stator inductance values.

The present paper starts by reviewing the different kinds of winding distributions: the properties of integralslot and fractional-slot balanced windings are described: the notion of circularity index, necessary for correct characterization of windings for machine with more than three phases, is introduced. The classical winding theory is then introduced as one of the most common way of modeling the winding distribution effects on the stator rotating field. The original matrix modeling is then described from the sampling of the winding function. Finally, it is shown that the matrix approach is a concise way of calculating the winding factors and estimating the set of self and mutual stator inductances of the multi-phase machine (taking into account space harmonics). Throughout the paper, two examples of existing five-phase machines, one being a prototype and the other being an industrial machine for an automotive application, will be considered in order to show the differences existing between their windings.

\section{Balanced multi-phase windings}

\subsection{Rules for obtaining a balanced multi-phase winding}

A balanced multi-phase winding is firstly characterised by the fact that, given the winding of phase number $k$, it is possible to deduce the winding of the following phase numbered $k+1$ by applying an angular spatial shift $\chi$. This condition is necessary but not sufficient in order to define

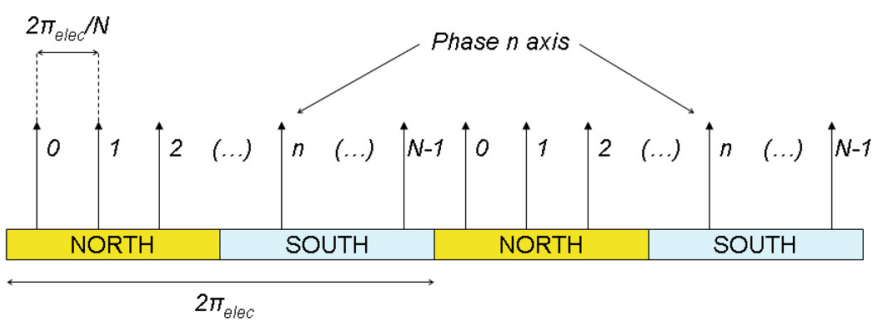

Fig. 1. (Color online) Representation of a balanced multiphase winding distribution $\left(\pi_{e l e c}=\frac{\pi}{p}, p\right.$ the pole pair number, $p=2$ in the figure).

a balanced winding for a $N$-phase rotating machine. It is also necessary to fullfill a property linked to the cyclicity of the machine: the angular shift between the last phase (numbered $N-1$ ) and the first phase (numbered 0) must also be equal to $\chi$. These two conditions imply that the angular spatial shift $\chi$ must verify:

$$
N \chi=2 \pi \frac{q}{r} \quad(q, r) \in \mathbb{Z}^{2} .
$$

Relation (1) implies that the angular spatial shift $\chi$ between two consecutive phases is generally $2 \pi / N$ modulated by a fractional number where $q$ denotes the numerator and $r$ denotes the denominator.

This notion of balance is illustrated by Figure 1. In this figure, each arrow symbolizes the position of a phase axis. The position of a phase axis depends on the arrangement of the coils that form the phase winding. The multi-phase winding is balanced if the phase axes are regularly distributed along the stator periphery as illustrated by Figure 1. From a practical point of view, the synthesis of a balanced multi-phase winding would be a simple task if the coils of the phase could be located anywhere around the stator periphery. Unfortunately, for physical reasons, the conductors of the coils must be placed inside the stator slots, which implies constraints with respect to the placement of the coils.

In this paper, the considered machines have slots that are regularly shifted around the circumference of the stator. In this case, in order to ensure a balanced winding for a $N$-phase machine with $N_{s}$ slots, $N_{s}$ must be a multiple of $N$ [4]. This condition actually results from the necessity of obtaining regularly shifted phases. As a consequence, a new relation must be verified by $\chi$ :

$$
\chi=\frac{2 \pi}{N_{s}} i_{c} \quad i_{c} \in \mathbb{Z}
$$

In relation $(2), i_{c}$ is the number of slots that corresponds to the spatial shift $\chi$ between two successive phases: $i_{c}$ is called the circularity index. The circularity index $i_{c}$ can be positive or negative depending on the direction of the rotating field generated by the winding. The value of $i_{c}$ is then calculated from mathematical constraints concerning the rotating field properties: these constraints ensure that the winding generates a satisfactory rotating field especially for machines with more than three phases. These constraints will be explored later in the paper. 


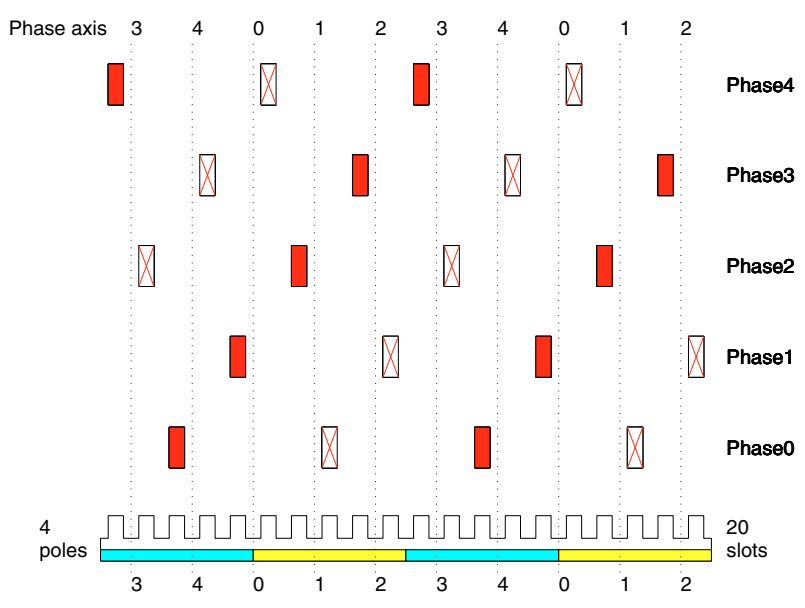

Fig. 2. (Color online) Example of a regular integral-slot balanced winding: case of a 5 -phase machine with 4 poles and 20 slots $\left(s_{p p}=1\right)$, the winding pattern is the same for each pole and each phase.

Let us consider $s_{p p}$, the number of slots per pole and per phase, commonly used for the characterization of windings:

$$
s_{p p}=\frac{N_{s}}{2 p N} .
$$

The two previous relations (1) and (2) imply the following property for the slot per phase and per pole number $s_{p p}$ of a balanced winding:

$$
s_{p p}=\frac{r i_{c}}{2 p q} .
$$

It appears that $s_{p p}$ is generally a fractional number. In the following paragraphs, two cases are distinguished depending on whether $s_{p p}$ is an integer or not.

\subsection{Integral-slot balanced windings}

According to [1], an integral-slot stator is one with an integral number of slots per pole, such that the pole-pitch is an integral number of slot-pitches, which means that $s_{p p}$ is an integer. The windings of integral-slot stators are naturally regular: the coils fall naturally into groups, with each group usually associated with one or two poles. Integral slot windings are widely used in asynchronous machines.

If the group is associated with one pole, the winding pattern is the same for each pole and each phase. Knowledge of the conductors distribution pattern above a pole allows the determination of the whole phase winding: these windings are said to be regular [31]. Figure 2 depicts the example of such a winding for a 5-phase machine with 20 slots and 4 poles: for each phase, the forward and backward conductors are represented. Each slot contains the conductors of a single phase. The winding is thus a single layer one. The winding is also fully-pitched in so far as the coil span is equal to the pole pitch.

If the group is associated with two poles, the winding is still balanced but irregular in so far as the winding pat-

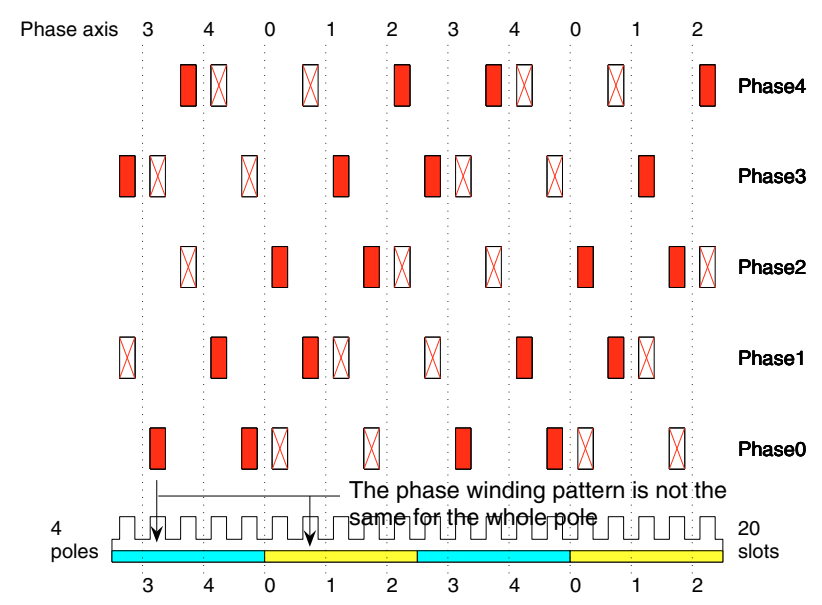

Fig. 3. (Color online) Example of an irregular integral-slot balanced winding with holes: case of a 5 -phase machine with 4 poles and 20 slots $\left(s_{p p}=1\right)$.



Fig. 4. (Color online) Example of an irregular balanced fractional winding for 5 -phase machine with 6 poles and 20 slots $\left(s_{p p}=2 / 3\right)$.

tern is not the same for each pole and each phase. In that case, the winding contains "holes" [31]. Figure 3 summarizes the 5 -phase 20 -slot 4 -pole configuration with such a winding. Since a slot contains conductors of two different phases, the winding is a double layer one. The described winding is not regular. If it had been regular, the second slot above the second pole (the 7th slot from the left of the figure) would have contained backward conductors of the first phase since the second slot above the first pole (the second slot from the left of the figure) contains forward conductors of the first phase. Nevertheless, as the $s_{p p}$ number is still an integer, the spatial winding period is equal to two pole pitches.

\subsection{Fractional-slot balanced windings}

When $s_{p p}$ is not an integer, the winding is necessarily irregular and is called a "fractional-slot balanced winding". Figure 4 gives the example of a fractional-slot winding for a 5 -phase machine with 20 slots and 6 poles. In this case, 
$s_{p p}$ is equal to $2 / 3$ and the winding is a double-layered one: each slot contains conductors of two different phases. As can be observed in Figure 4, there is no winding pattern above a pole or above a pole pair that could be reproduced to totally describe the winding. For the winding depicted in Figure 4, the distribution of the conductors inside all the stator slots must be observed in order to determine the winding pattern. Fractional-slot winding are often used in synchronous machines in order to reduce the reluctance torques [32].

\subsection{Reduced slot and pole pair numbers}

The previous sections illustrate the pattern notion for balanced multi-phase windings. This pattern actually corresponds to the spatial period of the phase winding: the spatial period can be quantified by the minimal number of slots to scan in order to totally characterize the distribution of the winding inside the slots. For a layout such that $s_{p p}$ is an integer (integral-slot configurations), the spatial period simply corresponds to two pole pitches (in Figs. 2 and 3, it is clear that the winding distribution is identically repeated above each pole pair). For the fractional-slot windings, the minimal number of slots to scan corresponds to an integer number of poles. This minimal number of slots is denoted $N_{s}^{\prime}$ and is expressed as follows:

$$
N_{s}^{\prime}=\frac{N_{s}}{\operatorname{gcd}\left(N_{s}, p\right)} .
$$

In this relation, $\operatorname{gcd}\left(N_{s}, p\right)$ is the greatest common divisor of $N_{s}$ and $p$. Relation (5) appears in [1] to define the spatial winding period: it is valid for every kind of balanced winding ( $s_{p p}$ integer or not). $N_{s}^{\prime}$ is called the reduced slot number. Similarly, the reduced pole pair number can be defined as:

$$
p^{\prime}=\frac{p}{\operatorname{gcd}\left(N_{s}, p\right)} .
$$

\subsection{Purpose of the circularity index}

The multi-phase windings demonstrate a circularity property that comes from the regular distribution of the stator slots. This property has already been taken into account using relation (2) which defines the circularity index $i_{c}$. The purpose of the circularity index is to attribute the $p^{\prime} N$ phase axes to the $N$ phases so that the rotating field generates $p$ pole pairs. The sign of the circularity index determines the direction of the rotating field (clockwise or counter-clockwise). For the examples of windings in Figures 2 and 3 , the phases are clockwise ordered and the circularity index is equal to 2 . Concerning the fractional winding in Figure 4, the circularity index is equal to 8. For a 3-phase machine, the circularity index is not really important in so far as the phases can only be clockwise or counter-clockwise. In other words, the stator rotating field generated by the alternative supply of the three phases always have the same pole number $(2 p)$ : it is just the direction of rotation of the rotating field (clockwise or counterclockwise) that can be different. It is not as simple for

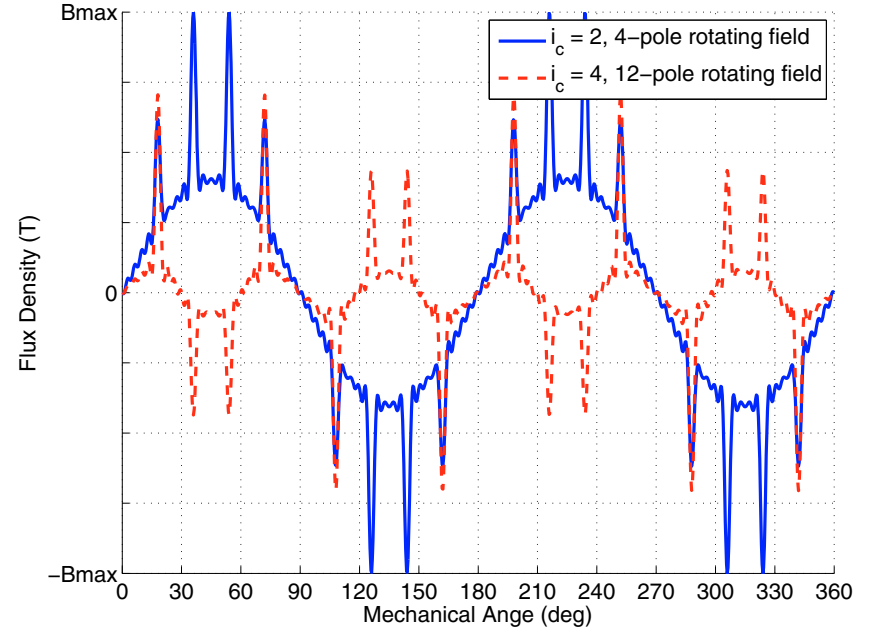

Fig. 5. (Color online) Comparison of the air gap flux density obtained with $i_{c}=2$ and $i_{c}=4$ for a 5 -phase machine (winding of Fig. 2 where $\left.\left(p, N_{s}\right)=(2,20)\right)$.

multi-phase machines. For example, for a five-phase PM machine where the pole number is imposed by the rotor magnet layout, the stator rotating field can have a number of main alternations three times higher than the rotor pole number if the phases are not correctly ordered. This fact is illustrated by Figure 5 which compares, for the fullypitched winding described in Figure 2, the field generated when the phase ordering corresponds to $i_{c}=2$ with the field generated when the phase ordering corresponds to $i_{c}=4$ in case of perfect sinusoidal current.

The purpose of the circularity index is then to correctly order the phases when the phase number is higher than three. We will see in the next paragraphs how this notion is used in order to obtain a matrix characterization of a winding.

\section{Common modeling using the winding function}

Used in [20], the winding function is a tool to model the influence of the winding on the stator rotating field. This function is extremely useful in order to separate, in the rotating field, the spatial contributions that are linked to the inherent characteristics of the winding machine, and the time contributions, that are linked to the supply of the machine. Consequently, the winding function allows us to study the adaptation of distribution of the coils inside the slots with this kind of supply.

\subsection{Definition of the winding function}

The definition of the winding function is based on the notion of stator magnetomotive force waves. These waves correspond to the stator rotating field that is measured in ampere-turns (AT). These waves are represented at an instant $t$ and for a position $\theta_{s}$ on the stator periphery. 


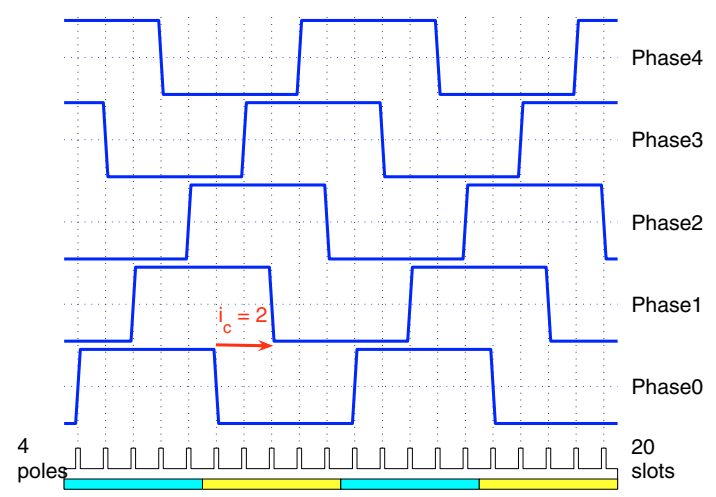

Fig. 6. (Color online) Winding function for the winding distribution described in Figure 2.

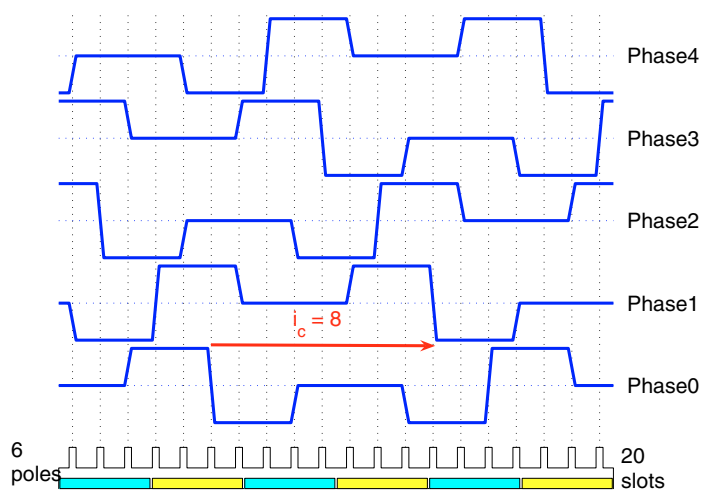

Fig. 7. (Color online) Winding function for the fractional winding distribution described in Figure 4.

The time variations result from the phase currents $i_{n}(t)$ whereas the spatial variations come from the distribution of the phase conductors inside the slots. This distribution for phase $n$ is modeled by the winding function $N_{n}\left(\theta_{s}\right)$. With these notations, the magnetomotive force wave can be expressed as:

$$
\mathcal{F}\left(\theta_{s}, t\right)=\sum_{n=0}^{N-1} N_{n}\left(\theta_{s}\right) i_{n}(t)
$$

In practical terms, the winding function at position $\theta_{s}$ is the sum of the conductors of phase $n$ that are inside the area $\left[0, \theta_{s}\right]$, calculated by positively counting the forward conductors and negatively counting the backward conductors. It corresponds to the magnetomotive force of a phase supplied by a constant current of one ampere. The unity of the winding function is the conductor-turn. Figures 6 and 7 give the winding function wave for the 5-phase winding described in Figures 2 and 4 respectively. The curves are normalized using their maximum. It can be observed that the winding function presents a linear variation of the conductor-turns along the slot opening. This information is obtained from the assumption that the slot conductors are uniformly spread along the slot opening. Consequently the winding function derivative is null everywhere except in the slot opening areas. The derivation of the winding function thus allows us to locate the phase conductors.

\subsection{Winding function derivative}

The derivative of the winding function defines the conductor density function $D_{n}\left(\theta_{s}\right)$ for a given phase $n$ :

$$
D_{n}\left(\theta_{s}\right)=\frac{1}{R_{s}} \frac{d N_{n}}{d \theta_{s}}
$$

In relation (8), $R_{s}$ is the air gap stator radius. The conductor density function indicates the number of phase conductors per meter for a point on the stator periphery, with the point being marked by the angle $\theta_{s}$. This function is notably used in [33]. It is not however defined as the winding function derivative.

\subsection{Winding function properties}

Figures 6 and 7 demonstrate two fundamental properties of the winding function:

- the zero average value;

- the circularity property between the different phases.

These two properties are also verified by its derivative function $D_{n}\left(\theta_{s}\right)$.

Around the stator, the number of forward conductors is equal to the number of backward conductors. Consequently the average value of the winding function is null:

$$
\int_{0}^{2 \pi} N\left(\theta_{s}\right) d \theta_{s}=0
$$

Of course, the winding function period cannot exceed $2 \pi$ (i.e. the total circumference of the stator). Relation (5) allows us to obtain a more precise value: the winding function period is equal to $2 \pi / \operatorname{gcd}\left(N_{s}, p\right)$ slot pitches. For example, concerning the integral-slot winding where $s_{p p}$ is an integer, the period is $2 \pi / p$ slot pitches (which actually correspond to two pole pitches) as shown by Figure 6 .

By assumption, the winding is balanced, which means that the phases are equivalent and regularly shifted by an angular shift $\chi$ according to relation (2). The circularity index $i_{c}$ is then introduced to deduce the winding of phase $\mathrm{n}$ from the winding function of the first phase.

$$
N_{n}\left(\theta_{s}\right)=N_{0}\left(\theta_{s}-n i_{c} \frac{2 \pi}{N_{s}}\right) .
$$

Properties (9) and (10) are also true for the conductor density function $D_{n}\left(\theta_{s}\right)$.

\subsection{Circularity index estimation}

By introducing the spatial Fourier series expansion of the winding function, the circularity index can be estimated:

$$
N_{0}\left(\theta_{s}\right)=\sum_{h=0}^{+\infty} \widehat{(N)}_{h} \cos \left(h \theta_{s}+\psi_{h}\right)
$$


Even though the winding function $N_{0}\left(\theta_{s}\right)$ is designed to generate $p$ pole pairs, it can be approximated by its spatial harmonic $p$ (by chosing the spatial frame such as $\psi_{p}=0$ ):

$$
N_{0}\left(\theta_{s}\right) \approx \widehat{(N)}_{p} \cos \left(p \theta_{s}\right)
$$

Relation (12) can be inserted into relation (10):

$$
N_{n}\left(\theta_{s}\right) \approx \widehat{(N)}_{p} \cos \left(p \theta_{s}-p n i_{c} \frac{2 \pi}{N_{s}}\right)
$$

As illustrated by Figure 5, the circularity index correctly orders the phases. This implies that winding functions $N_{n}\left(\theta_{s}\right)$ and $N_{n-1}\left(\theta_{s}\right)$ are spatially consecutive:

$$
\begin{gathered}
\left\{\begin{array}{l}
N_{n}\left(\theta_{s}\right) \approx \widehat{(N)}_{p} \cos \left(p \theta_{s}-p n i_{c} \frac{2 \pi}{N_{s}}\right) \\
N_{n-1}\left(\theta_{s}\right) \approx \widehat{(N)}_{p} \cos \left(p \theta_{s}-p(n-1) i_{c} \frac{2 \pi}{N_{s}}\right)
\end{array} \Rightarrow\right. \\
\begin{cases}p i_{c} \frac{2 \pi}{N_{s}}=\frac{2 \pi}{N}+k_{c} 2 \pi & \left(k_{c} \in \mathbb{Z}, \text { clockwise }\right) \\
p i_{c} \frac{2 \pi}{N_{s}}=-\frac{2 \pi}{N}+k_{c c} 2 \pi\left(k_{c c} \in \mathbb{Z},\right. & \text { counter-clockwise }) .\end{cases}
\end{gathered}
$$

The circularity index is deduced from relation (14). The implicit expressions give:

$$
\left\{\begin{array}{l}
i_{c} \in\left\{2 s_{p p}(1+N \mathbb{Z})\right\} \cap \mathbb{Z} \quad \text { (clockwise) } \\
i_{c} \in\left\{2 s_{p p}(-1+N \mathbb{Z})\right\} \cap \mathbb{Z} \text { (counter-clockwise). }
\end{array}\right.
$$

Relation (15) can be used to calculate the circularity index of the two windings described in this paper:

- for the integral-slot balanced winding of Figure 2 where $\left(N, p, N_{s}\right)=(5,2,20)$ and $s_{p p}=1$, relation $(15)$ yields $i_{c}=2$ for the clockwise order $\left(k_{c}=0\right.$ convenient in (14));

- for the fractional-slot balanced winding of Figure 4 where $\left(N, p, N_{s}\right)=(5,3,20)$ and $s_{p p}=2 / 3$, relation (15) yields $i_{c}=8$ for the clockwise order $\left(k_{c}=1\right.$ convenient in (14)).

\section{Matrix modeling of the multi-phase windings}

By definition, the winding function results from the stator magnetomotive force. Consequently this function is continous. However the discrete nature of the winding, imposed by the slots, yields a discrete modeling of the winding function. This section describes a matrix modeling of the machine winding that is based on the introduction of two matrices: the winding function matrix and the winding distribution matrix.

\subsection{Winding function matrix}

The winding function matrix consists of $N$ column vectors of $N_{s}$ components: component $k$ of vector $n$ corresponds to the value of the winding function at the right of slot $k$. The vectors are composed of the samples of the winding function at the slot level. This approach is close to that adopted in the discrete modeling of the field described in $[34,35]$ : in these papers, the matrix is composed of coefficients that result from the sampling of the air gap flux density under each tooth.

Mathematically two vectorial spaces are necessary to describe this winding function matrix:

- the first vectorial space $\mathcal{E}^{\mathcal{N}}$ has a dimension equal to the phase number $N$ and is associated with a canonic orthonormal basis

$$
\mathbf{B}^{\mathbf{N}}=\left\{\overrightarrow{n_{0}^{N}} \ldots \overrightarrow{n_{n}^{N}} \ldots \overrightarrow{n_{N-1}^{N}}\right\}
$$

- the second vectorial space $\mathcal{E}^{\mathcal{N}}$ has a dimension equal to the slot number $N_{s}$ and is associated with a canonic orthonormal basis

$$
\mathbf{B}^{\mathbf{N}_{\mathrm{s}}}=\left\{\overrightarrow{n_{0} N_{s}} \ldots \overrightarrow{n_{n}^{N_{s}}} \ldots \overrightarrow{n_{N_{s}-1} N_{s}}\right\}
$$

The winding function matrix is denoted $\underline{\underline{W}}$. The associated linear application is denoted $\mathcal{W}$ :

$\underline{\underline{W}}=\operatorname{mat}\left(\mathcal{W}, \mathbf{B}^{\mathbf{N}}, \mathbf{B}^{\mathbf{N}_{\mathbf{s}}}\right)=\left(w_{m, n}\right)_{(m, n) \in[0 \ldots N-1] \times\left[0 \ldots N_{s}-1\right]}$.

The column vector of $\underline{\underline{W}}$ is the sampling winding function for each phase and is denoted as follows:

$$
\underline{\underline{W}}=\left(\overrightarrow{w_{0}^{N_{s}}} \ldots \overrightarrow{w_{n}^{N_{s}}} \ldots \overrightarrow{w_{N-1}^{N_{s}}}\right)
$$

As the winding function matrix is built by sampling the continuous winding function, this matrix has the same properties:

- the "zero mean average property":

$$
\sum_{m=0}^{N_{s}-1} w_{m, n}=0
$$

- the "circularity property":

$$
w_{m, n}=w_{m-n i_{c}, 0} .
$$

Knowledge of the winding vector for the phase $n \overrightarrow{w_{n} N_{s}}$ (i.e. knowledge of the samples of the winding function for the phase $n$ ) allows us to rebuild the winding function:

$$
N_{n}\left(\theta_{s}\right) \approx n_{c d} \sum_{m=0}^{N_{s}-1} w_{m, n} S_{2 \pi / N_{s}}\left(\theta_{s}-m \frac{2 \pi}{N_{s}}\right) .
$$

In relation (20), $n_{c d}$ is the number of conductors inside a slot (under the assumption that all the slots have the same number of conductors) and $S_{2 \pi / N_{s}}$ is a periodic step function defined as follows:

$$
S_{\tau}(\theta)=\left\{\begin{array}{l}
1 \text { if } \theta \in[k 2 \pi, k 2 \pi+\tau], k \in \mathbb{Z} \\
0 \text { otherwise. }
\end{array}\right.
$$


In relation $(20)$, the $\approx$ operator denotes the loss of information due to the discrete integration of the samples of $N_{n}\left(\theta_{s}\right)$ : rigorously, along the slot opening, the winding function variation is linear (as can be shown in Figs. 6 and 7$)$. Rebuilding $N_{n}\left(\theta_{s}\right)$ removes this characteristic. The quantity of information lost when using this sampling method becomes more and more significant the larger the slot opening.

For example, the winding function matrix corresponding to the fractional winding described in Figure 7 is:

$$
\underline{\underline{W}}=\left(\begin{array}{ccccc}
0 & -1 / 2 & 1 / 2 & 1 / 2 & 0 \\
0 & -1 / 2 & -1 / 2 & 1 / 2 & 0 \\
1 / 2 & -1 / 2 & -1 / 2 & 0 & 0 \\
1 / 2 & 1 / 2 & -1 / 2 & 0 & 0 \\
1 / 2 & 1 / 2 & 0 & 0 & -1 / 2 \\
-1 / 2 & 1 / 2 & 0 & 0 & -1 / 2 \\
-1 / 2 & 0 & 0 & 1 / 2 & -1 / 2 \\
-1 / 2 & 0 & 0 & 1 / 2 & 1 / 2 \\
0 & 0 & -1 / 2 & 1 / 2 & 1 / 2 \\
0 & 0 & -1 / 2 & -1 / 2 & 1 / 2 \\
0 & 1 / 2 & -1 / 2 & -1 / 2 & 0 \\
0 & 1 / 2 & 1 / 2 & -1 / 2 & 0 \\
-1 / 2 & 1 / 2 & 1 / 2 & 0 & 0 \\
-1 / 2 & -1 / 2 & 1 / 2 & 0 & 0 \\
-1 / 2 & -1 / 2 & 0 & 0 & 1 / 2 \\
1 / 2 & -1 / 2 & 0 & 0 & 1 / 2 \\
1 / 2 & 0 & 0 & -1 / 2 & 1 / 2 \\
1 / 2 & 0 & 0 & -1 / 2 & -1 / 2 \\
0 & 0 & 1 / 2 & -1 / 2 & -1 / 2 \\
0 & 0 & 1 / 2 & 1 / 2 & -1 / 2
\end{array}\right) .
$$

The properties relative to the zero mean average and the circularity $\left(i_{c}=8\right)$ are clearly illustrated. This example also highlights the fact that the sampling is carried out at the right of the slots ${ }^{1}$, which means that the slope consecutive to the sum of the conductors along the slot opening is not taken into account.

\subsection{Distribution function matrix}

By adding for each phase the number of conductors around the stator circumference, the winding function is not really a design tool. In other words, knowledge of the winding function does not allow the direct identification of the described winding. In the context of the twodimensional model of the machine, the identification of

\footnotetext{
1 This is an arbitrary decision. It is possible to sample at the left of the slots.
}

the winding consists of attributing a phase to each slot conductor. For this purpose, a new tool is introduced: the distribution function matrix.

Each slot contains a number of conductors that can conduce the current in the positive direction (forward conductors) or in the negative direction (backward conductor). Each slot can contain a different number of conductors. The distribution function $\mathcal{D}$ distributes the phase conductors in the slots. $\mathcal{D}$ is then a linear application from the vectorial space $\mathcal{E}^{\mathcal{N}}$ to the vectorial space $\mathcal{E}^{\mathcal{N}}$. This linear application is represented by the matrix $\underline{\underline{D}}$ :

$$
\begin{aligned}
\underline{\underline{D}} & =\operatorname{mat}\left(\mathcal{D}, \mathbf{B}^{\mathbf{N}}, \mathbf{B}^{\mathbf{N}_{\mathbf{s}}}\right) \\
& =\left(d_{m, n}\right)_{(m, n) \in[0 \ldots N-1] \times\left[0 \ldots N_{s}-1\right]} .
\end{aligned}
$$

The column vectors of $\underline{\underline{D}}$ are the phase distribution vectors and are denoted as follows:

$$
\underline{\underline{D}}=\left(\overrightarrow{d_{0}{ }^{N_{s}}} \ldots \overrightarrow{d_{n}^{N_{s}}} \ldots \overrightarrow{d_{N-1} N_{s}}\right)
$$

By definition, the conductor proportion $d_{m, n}$ of the slot $m$ that is attributed to the phase $n$ is signed:

- if $d_{m, n}$ is positive, it is a proportion of the forward conductors;

- if $d_{m, n}$ is negative, it is a proportion of the backward conductors.

As the number of forward conductors is equal to the number of backward conductors, the sum of the vector $\overrightarrow{d_{n}{ }^{N}}$ is null:

$$
\sum_{g=0}^{N_{s}-1} d_{g, n}=0
$$

Furthermore the absolute value of the proportion of slot conductors attributed to the phase can not exceed one:

$$
\forall(m, n) \in\left[0 \ldots N_{s}-1\right] \times[0 \ldots N-1]-1 \leq d_{m, n} \leq 1 .
$$

Moreover, if all the conductors available in the slot $m$ are used, the sum of the absolute values of the conductor proportions for each phase is equal to 1 :

$$
\forall m \in\left[0 \ldots N_{s}-1\right] \sum_{n=0}^{N-1}\left|d_{m, n}\right|=1 .
$$

It is important to underline that this representation does not take into account the relative position of the conductors inside the slots (this already the case when building of the winding function). For example, for a slot that contains conductors of two different phases, the two standard conductor arrangements depicted by Figure 8 result in the same coefficients in matrix $\underline{\underline{D}}$. This simplification is necessary in the context of the analytical calculation of the flux density for a slotted machine [36]. Some examples of distribution function matrices for a 2-pole 3 -phase machine 


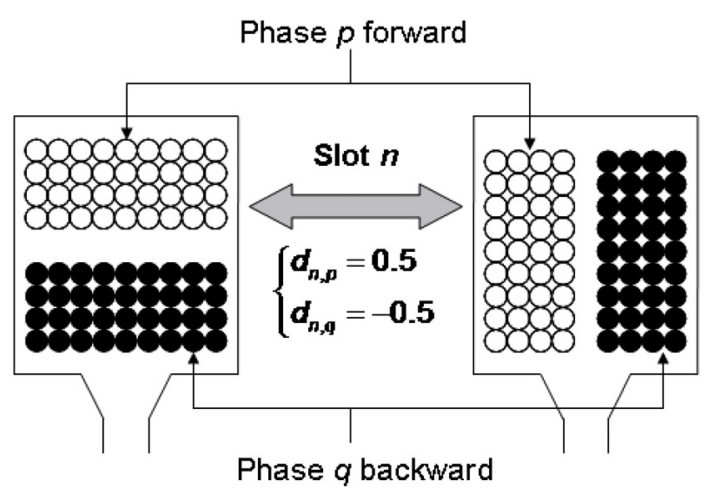

Fig. 8. Inability of the model to take into account the relative position of the conductors inside the slots.

with 6 slots are given:

$$
\begin{gathered}
\underline{\underline{D}}=\underbrace{\left(\begin{array}{ccc}
1 & 0 & 0 \\
-1 & 0 & 0 \\
0 & 1 & 0 \\
0 & -1 & 0 \\
0 & 0 & 1 \\
0 & 0 & -1
\end{array}\right)}_{\text {Short coils }}, \underline{\underline{D}}=\underbrace{\left(\begin{array}{ccc}
1 & 0 & 0 \\
0 & 0 & -1 \\
0 & 1 & 0 \\
-1 & 0 & 0 \\
0 & 0 & 1 \\
0 & -1 & 0
\end{array}\right)}_{\text {Long coils }}, \\
\underline{\underline{D}}=\underbrace{\left(\begin{array}{ccc}
0 & -0.5 & 0.5 \\
0.5 & 0 & -0.5 \\
0.5 & 0 & -0.5 \\
-0.5 & 0.5 & 0 \\
-0.5 & 0.5 & 0 \\
0 & -0.5 & 0.5
\end{array}\right)}_{\text {Two-layer winding }}
\end{gathered}
$$

It can be noticed that these three distribution function matrices verify relations (25), (26) and (27).

Finally, the distribution function matrix can be considered as a natural reading of the phase winding: this matrix can be directly deduced from a simple representation like the ones in Figures 2-4. Nevertheless, it must be remembered that the distribution function does not specify the way to connect the conductors in order to make the coils. This knowledge is thus not sufficient to fully characterize the winding, despite being nonetheless necessary.

\subsection{Mathematical link between winding and distribution function matrices}

As the discrete winding function sums the slot conductors for each phase and the conductor distribution function locates the conductors for a given phase, a relation of discrete derivation allows us to determine $\underline{\underline{D}}$ from $\underline{\underline{B}}$. In other words, the components of the vector $\overrightarrow{d_{n}{ }^{N_{s}}}$ can be deduced from the ones of the vector $\overrightarrow{w_{n}{ }^{N_{s}}}$ :

$$
\forall m \in[1 \ldots N-1] d_{m, n}=w_{m, n}-w_{m-1, n} .
$$

If the example of Figures 4 and 7 is considered, the matrix $\underline{\underline{D}}$ is directly obtained by using relation (28) on the matrix $\underline{\underline{W}}$ of relation $(22)$ :

$$
\underline{\underline{D}}=\left(\begin{array}{ccccc}
0 & -1 / 2 & 0 & 0 & 1 / 2 \\
0 & 0 & -1 & 0 & 0 \\
1 / 2 & 0 & 0 & -1 / 2 & 0 \\
0 & 1 & 0 & 0 & 0 \\
0 & 0 & 1 / 2 & 0 & -1 / 2 \\
-1 & 0 & 0 & 0 & 0 \\
0 & -1 / 2 & 0 & 1 / 2 & 0 \\
0 & 0 & 0 & 0 & 1 \\
1 / 2 & 0 & -1 / 2 & 0 & 0 \\
0 & 0 & 0 & -1 & 0 \\
0 & 1 / 2 & 0 & 0 & -1 / 2 \\
0 & 0 & 1 & 0 & 0 \\
-1 / 2 & 0 & 0 & 1 / 2 & 0 \\
0 & -1 & 0 & 0 & 0 \\
0 & 0 & -1 / 2 & 0 & 1 / 2 \\
1 & 0 & 0 & 0 & 0 \\
0 & 1 / 2 & 0 & -1 / 2 & 0 \\
0 & 0 & 0 & 0 & -1 \\
-1 / 2 & 0 & 1 / 2 & 0 & 0 \\
0 & 0 & 0 & 1 & 0
\end{array}\right) .
$$

The concordance between this matrix $\underline{\underline{D}}$ and the representation adopted in Figure 4 is well illustrated: for the first slot, one half of the slot conductors is backward for the second phase and the other half is forward for the fifth phase.

A discrete integration relation allows us to deduce the winding function matrix from the distribution function matrix. As required by relation (18), the integration constant must be chosen to cancel the sum of the components of the winding function vectors. The following relation can be derived:

$$
\begin{aligned}
\forall(m, n) \in\left[0 \ldots N_{s}-1\right] & \times[0 \ldots N-1] \\
w_{m, n} & =\sum_{g=0}^{m} d_{g, n}-\frac{1}{N_{s}} \sum_{k=0}^{N_{s}-1} \sum_{h=0}^{k} d_{h, n} .
\end{aligned}
$$

This discrete derivative relation going to the distribution function matrix $\underline{\underline{D}}$ from the winding function matrix $\underline{\underline{W}}$ is similar to the derivative relation (8) that defines the function of conductor density $D_{n}\left(\theta_{s}\right)$. It is clear that this function $D_{n}\left(\theta_{s}\right)$ can be built with the components of the phase distribution vector $\overrightarrow{d_{n} N_{s}}$. Under the assumption 
that each slot contains the same number of conductors $n_{c d}$, the following relation can be written (where $\sigma_{s}$ is the slot-opening in meters):

$$
D_{n}\left(\theta_{s}\right)=\frac{n_{c d}}{R_{s} \sigma_{s}} \sum_{m=0}^{N_{s}-1} d_{m, n} S_{\sigma_{s}}\left(\theta_{s}-\frac{\pi}{N_{s}}-m \frac{2 \pi}{N_{s}}\right)
$$

In opposition to relation (20), which approximates the winding function $N_{n}\left(\theta_{s}\right)$, relation (31) gives the exact function of the conductor density $D_{n}\left(\theta_{s}\right)$. Actually the function of conductor density is a linear combination of step signals with a step equal to the slot opening $\sigma_{s}$. The matrix $\underline{\underline{D}}$ identifies for each phase the coefficients of the linear combination. By indicating for each slot the proportion of conductors that is attributed to a phase, the function of the conductor density is more significative for the designer than the winding function.

\section{Use of the winding matrix modeling to directly characterize the winding factors and the mutual inductances}

\subsection{Link between the phase distribution vector components and the usual winding factors}

The proposed approach is similar to the EMF phasor approach $[3,6]$ and is based on the introduction of the flux per slot. This flux per slot corresponds to the flux density on the stator periphery integrated along the slot opening for each conductor inside the slot. For the first slot (numbered 0), this flux per slot can be defined as follows:

$$
\phi_{s, 0}(\theta)=\int_{\gamma=0}^{2 \pi} A(\theta, \gamma) S_{\tau_{s}}\left(\gamma-\frac{\pi}{N_{s}}\right) R_{s} d \gamma
$$

From relation (32), $\theta$ is the rotor position and $A(\theta, \gamma)$ is the potential vector in the stator periphery (i.e. at the radius $R_{s}$ ) for the rotor position $\theta$ estimated at the angle $\gamma$ in the stator frame. If the flux density source is the rotor, the flux per slot is necessarily $2 \pi / p$-periodic and can be expanded using Fourier series:

$$
\phi_{s, 0}(\theta)=\sum_{h=-\infty}^{+\infty} \widehat{\left(\phi_{s}\right)} e^{j h p \theta}
$$

The flux per slot for the slot numbered $m$ is obtained by shifting the flux per slot of the first slot. Its expression can thus be deduced from (32) and its Fourier expansion from (33):

$$
\begin{aligned}
\phi_{s, m}(\theta) & =\phi_{s, 0}\left(\theta-m \frac{2 \pi}{N_{s}}\right) \\
& =\sum_{h=-\infty}^{+\infty} \widehat{\left(\phi_{s}\right)_{h}} e^{-j \frac{2 \pi}{N_{s}} h m} e^{j h p \theta}
\end{aligned}
$$

If the effects of the end-turns are neglected, the flux per phase $\phi_{n}(\theta)$ can be expressed by summing the flux per slots rated by the winding distribution coefficients:

$$
\phi_{n}(\theta)=\sum_{m=0}^{N_{s}-1} d_{m, n} \phi_{s, m}(\theta)
$$

By combining relations (35) and (34), the Fourier expansion of the flux per phase is given by:

$$
\phi_{n}(\theta)=\sum_{h=-\infty}^{+\infty} \widehat{\left(\phi_{s}\right)_{h}}\left(\sum_{m=0}^{N_{s}-1} d_{m, n} e^{-j \frac{2 \pi}{N_{s}} m p h}\right) e^{j h p \theta} .
$$

In relation (36), the term inside the brackets shows how the phase distribution coefficients act on the spectrum of the flux per phase. Actually this term corresponds to the coil disposition factor $K_{d, h}$ :

$$
K_{d, h}=\left|\sum_{m=0}^{N_{s}-1} d_{m, n} e^{-j \frac{2 \pi}{N_{s}} m p h}\right| .
$$

By definition, the winding factor is the ratio of flux linked by an actual winding to flux that would have been linked by a fully-pitched concentrated winding with the same number of turns. It is well-known that this configuration leads to the highest coil disposition factor. As shown in Appendix A, the winding factor of any winding can be deduced from the coil disposition factor expression (37):

$$
K_{h}=\frac{N}{N_{s}} K_{d, h}=\frac{N}{N_{s}}\left|\sum_{m=0}^{N_{s}-1} d_{m, n} e^{-j \frac{2 \pi}{N_{s}} m p h}\right| .
$$

The method of obtaining the winding factor is compatible with the more common method that consists in calculating the winding factor by using the distribution factor and the pitch factor as in [37].

The final expression (38) is a very concise mean of calculating the winding factors and subsequently predicting the influence of the winding on the electromotive force spectrum. For the example of the fractional winding depicted in Figure 4 where $\left(N_{s}, p, N\right)=(20,3,5)$, the third harmonic winding factor can be easily estimated via relation (38) used for the winding distribution given in (29):

$$
K_{3}=\frac{5}{20}\left|\sum_{m=0}^{19} d_{m, 0} e^{j \frac{\pi}{10} 2 \times 3}\right| \approx 0.794
$$

\subsection{Estimation of the air gap mutual inductance values from the winding function matrix}

For a slotted machine, the supply of a phase generates two kinds of bidimensionnal flux paths:

- the flux paths that cross the air gap whose effects are modeled by the air gap mutual inductances;

- the flux paths that do not cross the air gap whose effects are modeled by the leakage mutual inductances. 
The winding function theory enables the estimation of the air gap mutual inductance values with the assumption of radial path for the air gap flux density. It is then possible to directly deduce the air gap flux density from the winding function. If the phase numbered 0 is supplied with a constant current $I_{0}$, the air gap flux density given by:

$$
B(\gamma) \approx \frac{\mu_{0}}{g+g_{m}} N_{0}(\gamma) I_{0}
$$

The flux per phase variation $d \phi_{n}$ can be expressed as the product of the phase winding function with the flux density linked by a conductor:

$$
d \phi_{n}=N_{n}(\gamma) B(\gamma) L_{m} R_{s} d \gamma
$$

If relation (40) is used, expression (41) becomes:

$$
\phi_{n}=\frac{\mu_{0}}{g+g_{m}} L_{m} R_{s} I_{0} \int_{\gamma=0}^{2 \pi} N_{0}(\gamma) N_{n}(\gamma) d \gamma .
$$

If expression (20), which rebuilds the winding function from its sample is introduced, the flux per phase is:

$$
\phi_{n}=I_{0} \underbrace{\frac{\mu_{0}}{g+g_{m}} L_{m} R_{s} n_{c d}^{2} \frac{2 \pi}{N_{s}}}_{=C_{m^{a}}} \sum_{m=0}^{N_{s}-1} w_{m, 0} w_{m, n} .
$$

The air gap mutual inductance $m_{n}^{a}$ that represents the air gap flux density collected by the phase $n$ when the phase 0 is supplied is then very simply estimated. It is proportional to the dot product of the phase $n$ winding function vector with the phase 0 winding function vector:

$$
m_{n}^{a}=C_{m^{a}} \overrightarrow{w_{0}^{N_{s}}} \cdot \overrightarrow{w_{n}^{N_{s}}} .
$$

The air gap self-inductance of a phase is $m_{0}^{a}$. The coefficient $C_{m^{a}}$ is mainly relative to the geometrical characteristics of the machine.

The relation (44) allows us to directly obtain the stator matrix air gap inductance $\underline{\underline{M_{s s}^{a}}}$ with the winding function matrix $\underline{\underline{W}}$ :

$$
\underline{\underline{M_{s s}^{a}}}=C_{m^{a}} \underline{\underline{W^{T}}} \underline{\underline{W}}
$$

Relation (45) can also be obtained by considering the magnetic energy stored in the air gap as in [34].

\subsection{Estimation of the leakage mutual inductance values from the distribution function matrix}

As stated previously, the leakage inductances result from the flux density that does not cross the air gap. The leakage self inductance $m_{0}^{l}$ corresponds to the outside air gap flux density linked by the phase that is supplied. The leakage mutual inductance $m_{n}^{l}$ corresponds to the outside air gap flux density linked by the phase numbered $n-1$ when the phase numbered 0 is supplied. The common analytical methods do not allow the calculation of the flux density around the slots. Consequently additional assumptions concerning these flux paths are necessary.

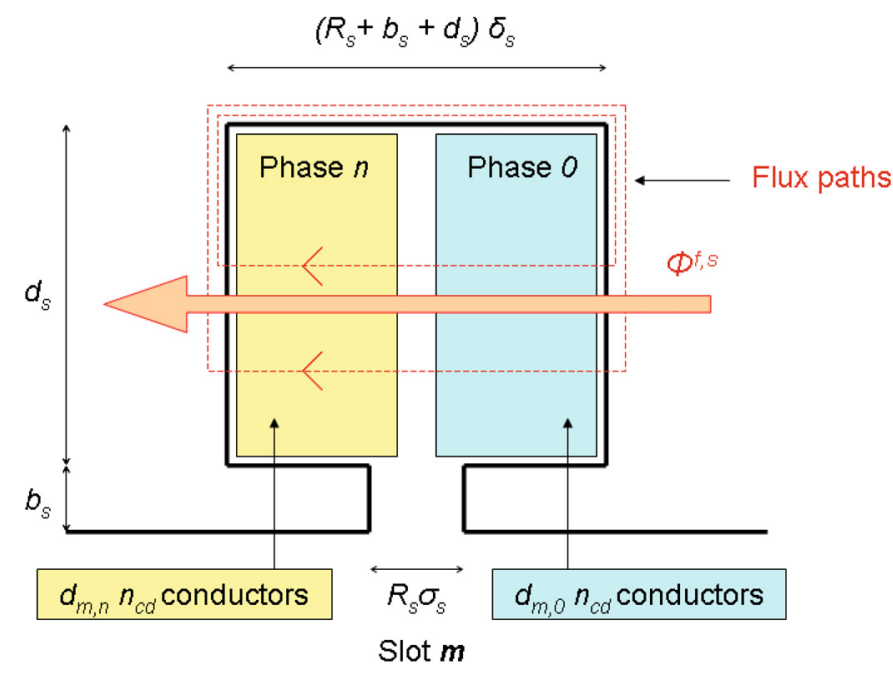

Fig. 9. (Color online) Assumptions for the flux paths inside the iron to estimate the leakage inductance of a slot.

In [38], several analytical methods to estimate the leakage flux for various slot geometries are described. These methods are based on the following assumptions:

- the iron permeability is infinite;

- the flux paths inside the slots are considered perpendicular to the slot sides and are closed up in the iron core.

In this reference, only the case where the slot contains conductors of a single phase is presented. It is possible to extend this approach to the case of multi-layer windings. For this paper, we limit the description to the case depicted on the right part of Figure 8 that corresponds to the conductor disposition of the machine M5-B. For the case described on the left part, a similar method to the one described subsequently can be used.

Figure 9 illustrates the hypothesis concerning the flux path inside the slots. For this example, the slot numbered $m$ contains two conductors areas: the first belongs to the phase 0 and the second belongs to the phase numbered $m$. The $d_{m, 0} n_{c d}$ conductors of phase 0 are considered to be supplied with a continuous current of magnitude $I_{0}$ : these supplied conductors generate the flux density. The resulting flux path cross the slot and are closed up in the iron core. Each slot conductor, regardless of its belonging phase, cuts a flux $\phi^{l, s}$. This flux can be calculated by using the Ampere theorem for every contour path then by integrating the resulting flux densities on the conductor area. For the considered slot geometry, if phase 0 is supplied, the flux density collected by a conductor is:

$$
\phi^{l, s}=\mu_{0} d_{m, 0} n_{c d} I_{0}\left(\frac{p_{s}}{3\left(R_{s}+b_{s}+p_{s}\right) \delta_{s}}+\frac{b_{s}}{R_{s} \sigma_{s}}\right) .
$$

In order to estimate the total flux linked by the conductors of phase $n$ located in the slot numbered $m$, the flux $\phi^{l, s}$ 
must be multiplied by the conductor number $d_{m, n} n_{c d}$ :

$$
\begin{aligned}
\Phi_{n}^{f, s} & =d_{m, n} n_{c d} \phi^{l, s} \\
& =\mu_{0} d_{m, n} d_{m, 0} n_{c d}^{2} I_{0}\left(\frac{p_{s}}{3\left(R_{s}+b_{s}+p_{s}\right) \delta_{s}}+\frac{b_{s}}{R_{s} \sigma_{s}}\right) .
\end{aligned}
$$

The leakage mutual inductance $m_{n}^{l}$ is then obtained by summing the leakage fluxes $\Phi_{n}^{l, s}$ of each slot and by dividing by the constant current $I_{0}$ :

$$
\begin{aligned}
m_{n}^{l}= & \underbrace{\mu_{0} n_{c d}^{2}\left(\frac{p_{s}}{3\left(R_{s}+b_{s}+p_{s}\right) \delta_{s}}+\frac{b_{s}}{R_{s} \sigma_{s}}\right)}_{=K_{m} l} \sum_{m=0}^{N_{s}-1} d_{m, n} d_{m, 0} .
\end{aligned}
$$

In relation (48), $K_{m^{l}}$ is a quantity homogeneous to an inductance and only depends on the slot geometry. In other words, the value of $K_{m^{l}}$ results from the integration of the flux density inside the slot. If the slot geometry is then changed, only $K_{m^{l}}$ has to be changed. The second part of relation (48) only depends on the winding distribution. As for equation (44), which gives the mutual air gap inductance, the mutual leakage inductance can be viewed as a dot product:

$$
m_{n}^{l}=K_{m^{l}} \overrightarrow{d_{n} N_{s}} \cdot \overrightarrow{d_{0} N_{s}} .
$$

Relation (49) shows that the mutual leakage inductance mainly relates to the dot product of the winding distribution vectors. Common results can be found with relation (49). For the example of a single layer winding, as the slots contain conductors of a single phase, the winding distribution vectors are orthogonal to each other. According to relation (49), the mutual leakage inductances are null, which is a well-known result (for conventional machines where the slots are not too closed each other).

As for relation (45) related to the air gap mutual inductance matrix, it is possible to introduce the leakage inductance matrix from relation (49):

$$
\underline{\underline{M_{s s}^{l}}}=K_{m^{l}} \underline{\underline{D}}^{T} \underline{\underline{D}} .
$$

The stator inductance matrix is then obtained by summing relations (45) and (50):

$$
\underline{\underline{M_{s s}}}=C_{m^{a}} \underline{\underline{W^{T}}} \underline{\underline{W}}+K_{m^{l}} \underline{\underline{D}}^{T} \underline{\underline{D}} .
$$

\subsection{Validation}

In order to validate the analytical expressions of the inductances, two 5-phase real machines are used. The main parameters of these machines are given in Tables 1 and 2 . The first machine, called "M5-A", has a classical single layer fully pole-pitched winding $\left(s_{p p}=1\right.$, see Figs. 2

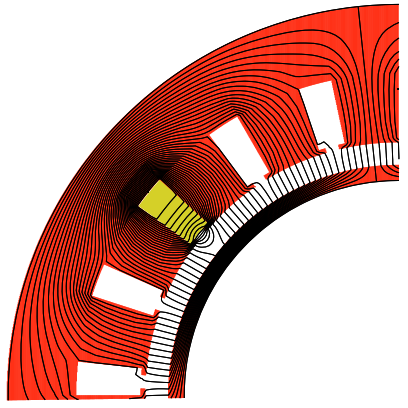

Fig. 10. (Color online) Flux path for M5-A machine $\left(s_{p p}=1\right)$ where a single phase is supplied.

Table 1. Main characteristics of the machine M5-A $\left(s_{p p}=1\right)$.

\begin{tabular}{c|c}
\hline & \\
M5-A & $1.5 \mathrm{~kW}$ \\
Power & $\approx 10 \mathrm{~N} \mathrm{~m}$ \\
Torque & $1500 \mathrm{rpm}$ \\
Speed & 5 \\
Phase number $N$ & 20 \\
Slot number $N_{s}$ & 4 \\
Pole number $2 p$ & Internal \\
Rotor Type & $80 \mathrm{~mm}$ \\
\hline Effective length $L_{m}$ & $15 \mathrm{~mm}$ \\
Stator yoke thickness $e_{c s}$ & $55 \mathrm{~mm}$ \\
Stator radius $R_{s}$ & $1.5 \mathrm{~mm}$ \\
Mechanical air gap $g_{m}$ & $3.4 \mathrm{~mm}$ \\
Magnet layer thickness $g_{a}$ & $1.07 \mathrm{~T}$ \\
Remanent flux density $B_{r}$ & $100 \%$ \\
Magnet arc to pole pitch ratio & $30 \mathrm{~mm}$ \\
Rotor yoke thickness $e_{c r}$ & $0.33 \tau_{s}$ \\
\hline Slot width $\delta_{s}\left(\tau_{s}\right.$, tooth pitch) & $0.5 \delta_{s}$ \\
Slot width opening $\sigma_{s}$ & $1 \mathrm{~mm}$ \\
Slot-closing thickness $b_{s}$ & $15 \mathrm{~mm}$ \\
Slot depth $p_{s}$ & 25 \\
Conductor number $n_{c d}$ & $1 \mathrm{~mm}{ }^{2}$ \\
Conductor section $s_{c d}$ & \\
\hline & \\
\hline & \\
\hline &
\end{tabular}

and 6) whereas the second one, called "M5-B", has a two layer fractional slot winding $\left(s_{p p}=2 / 3\right.$, see Figs. 4 and 7$)$. In order to assess the pertinence of the hypothesis relative to the flux path inside the slot, Figures 10 (machine M5A) and 11 (machine M5-B) present the flux path using the 2D numerical software Difimedi where a single phase is supplied.

First, for the two machines, the flux paths in the air gap are globally radial. Consequently, the hypothesis taken to write relation (40) and then to obtain final relation (44) (for air gap mutual inductance $m_{n}^{a}$ ) is correct. Figure 10 depicts the flux paths for M5-A machine: it can be seen that, for the slot containing the supplied conductors, the flux paths exclusively in the iron do not penetrate other slots, thus do not reach conductors of another phase. Consequently, for this machine, the common leakage flux is insignificant. On the contrary, for the M5-B machine 
Table 2. Main characteristics of the machine M5-B $\left(s_{p p}=\right.$ $2 / 3)$.

\begin{tabular}{c|c}
\hline & \\
M5-B & $0.3 \mathrm{~kW}$ \\
Power & $0.9 \mathrm{~N} \mathrm{~m}$ \\
Torque & $3500 \mathrm{rpm}$ \\
Speed & 5 \\
Phase number $N$ & 20 \\
Slot number $N_{s}$ & 6 \\
Pole number $2 p$ & External \\
Rotor type & $35 \mathrm{~mm}$ \\
\hline Effective length $L_{m}$ & $6 \mathrm{~mm}$ \\
Stator yoke thickness $e_{c s}$ & $1 \mathrm{~mm}$ \\
Stator radius $R_{s}$ & $4 \mathrm{~mm}$ \\
Mechanical air gap $g_{m}$ & $0.35 \mathrm{~T}$ \\
Magnet layer thickness $g_{a}$ & $97 \%$ \\
Remanent flux density $B_{r}$ & $3 \mathrm{~mm}$ \\
Magnet arc to pole pitch ratio & $0.75 \tau_{s}$ \\
Rotor yoke thickness $e_{c r}$ & $0.33 \delta_{s}$ \\
\hline Slot width $\delta_{s}$ & $1.5 \mathrm{~mm}$ \\
Slot width opening $\sigma_{s}$ & $14 \mathrm{~mm}$ \\
Slot-closing thickness $b_{s}$ & 40 \\
Slot depth $p_{s}$ & $0.5 \mathrm{~mm}{ }^{2}$ \\
Conductor number $n_{c d}$ & \\
Conductor section $s_{c d}$ & \\
\hline
\end{tabular}

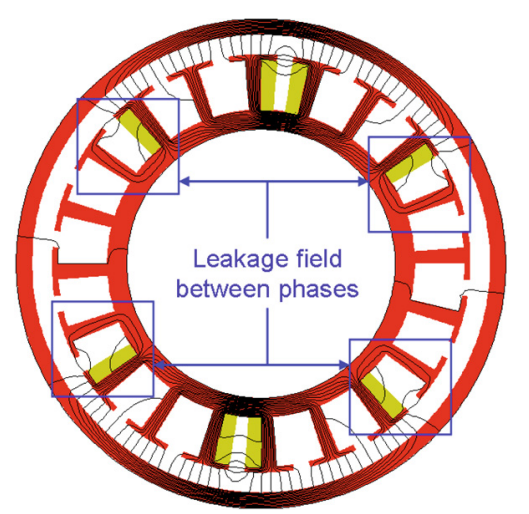

Fig. 11. (Color online) Flux path for M5-B machine $\left(s_{p p}=1\right)$ where a single phase is supplied.

with a two layer winding, Figure 11 shows the existence of mutual flux paths that do not cross the air gap: if a slot containing supplied conductors is considered, the flux lines reach the conductors of the other phase located in the same slot without crossing the air gap. Consequently, for the M5-B machine, the leakage mutual inductance is significant.

Tables 3 and 4 give the results obtained for the set of inductances. In order to point out the advantages of the analytical method described in this paper (relation (51), $\left.m_{n}=m_{n}^{a}+m_{n}^{l}\right)$, the tables start by giving the results obtained with the classical first harmonic approach. This common method is recalled in Appendix B, which con-
Table 3. Comparison of the inductance values obtained for M5-A machine.

\begin{tabular}{c|ccc}
\hline Method & $m_{0}(\mathrm{mH})$ & $m_{1}(\mathrm{mH})$ & $m_{2}(\mathrm{mH})$ \\
\hline Analytical (B.1) & 1.02 & 0.32 & -0.82 \\
Analytical (B.2) & 1.28 & 0.32 & -0.82 \\
Analytical (51) & 1.28 & 0.20 & -0.61 \\
Numerical & 1.37 & 0.20 & -0.60 \\
Measurement & 1.31 & 0.20 & -0.52 \\
\hline
\end{tabular}

Table 4. Comparison of the inductance values obtained for M5-B machine.

\begin{tabular}{c|ccc}
\hline Method & $m_{0}(\mathrm{mH})$ & $m_{1}(\mathrm{mH})$ & $m_{2}(\mathrm{mH})$ \\
\hline Analytical (B.1) & 0.62 & 0.19 & -0.50 \\
Analytical (B.2) & 0.75 & 0.19 & -0.50 \\
Analytical (51) & 0.75 & 0 & -0.23 \\
Numerical & 0.89 & 0.01 & -0.26 \\
Measurement & 1.13 & 0.03 & -0.17 \\
\hline
\end{tabular}

cludes by giving two relations to value the inductances: relation (B.1) is dedicated to the air gap inductances $m_{n}^{a}$ whereas relation (B.2) integrates leakage effects and estimates the inductances $m_{n}$. The two tables also give the values $m_{n}$ obtained with the numerical software and the measured values. Appendix $\mathrm{C}$ indicates the method chosen to measure the set of inductances.

The new analytical method differs from the first harmonic approach for the two following reasons:

- all the magnetomotive force harmonics are taken into account;

- the possible leakage mutualizations are modeled.

With reference to the numerical calculations, Tables 3 and 4 clearly show the precision improvement obtained with the new analytical method. The case of the M5-B machine is particularly demonstrative because its fractionalslot winding implies a great deal of spatial harmonics and a leakage mutualization between phases (as shown by Fig. 11). Table 4 clearly shows that first harmonic approach is not adapted to estimate the inductances: the new relation (51) allows us to obtain more accurated results.

However the values obtained by measurements temper this conclusion since they are not so closed to the numerical ones. As a consequence, at this level it must be pointed out that the main interest of the described approach in the paper is to give practical algorithms for numerical implementation.

To better understand the necessity of the proposed approach for machines with more than three phases, it is necessary to examine not only mutual inductances but also the synchronous inductances. If for a wye-coupled three-phase machine only one synchronous inductance characterizes the machine, three synchronous inductances denoted $L_{M 1}, L_{M 2}$ and $L_{H}$ are found for a five-phase machine [25]. If $L_{M 1}$ is associated with the first harmonic, $L_{M 2}$ and $L_{H}$ depend on other harmonics. As $L_{M 2}$ and $L_{H}$ directly impact the choice of the PWM frequency of the VSI that supplies the machine, it is necessary to get 
Table 5. Comparison of the synchronous inductance values obtained for the M5-A machine.

\begin{tabular}{c|ccc}
\hline Method & $L_{H}(\mathrm{mH})$ & $L_{M 1}(\mathrm{mH})$ & $L_{M 2}(\mathrm{mH})$ \\
\hline Ana. (B.1) & 0 & 2.55 & 0 \\
Ana. (B.2) & 0.26 & 2.81 & 0.26 \\
Ana. (51) & 0.46 & 2.39 & 0.57 \\
Numerical & 0.56 & 2.47 & 0.67 \\
Measurement & 0.67 & 2.27 & 0.67 \\
\hline
\end{tabular}

Table 6. Comparison of the synchronous inductance values obtained for the M5-B machine.

\begin{tabular}{c|ccc}
\hline Method & $L_{H}(\mathrm{mH})$ & $L_{M 1}(\mathrm{mH})$ & $L_{M 2}(\mathrm{mH})$ \\
\hline Ana. (B.1) & 0 & 1.55 & 0 \\
Ana. (B.2) & 0.13 & 1.68 & 0.13 \\
Ana. (51) & 0.29 & 1.12 & 0.61 \\
Numerical & 0.39 & 1.32 & 0.70 \\
Measurement & 0.84 & 1.43 & 0.97 \\
\hline
\end{tabular}

correct values in order to design a machine adapted to the possibilities of the VSI supply.

Tables 5 and 6 present thus the synchronous inductances obtained from Tables 3 and 4 . For both machines the errors on synchronous inductances $L_{M 2}$ and $L_{H}$, which are associated with harmonics greater than the first harmonic, are clearly important if the classical first harmonic approach is used. On the contrary, the new relation (51) greatly improves the estimation: it is particularly obvious for the fractional-slot winding of M5-B machine (see Tab. 6). At this level it appears clearly that it was necessary to consider an approach, which is easy to implement for numerical calculations (using matrix approach) but also taking into account easily the spatial harmonics.

\section{Conclusion}

The matrix modeling of multi-phase winding described in this paper is adapted for rotating machines with a regular distribution of slots around the stator circumference. On the one hand, the simple method deducing the winding factor from one of the columns of the distribution matrix is valid for every kind of air gap. On the other hand, the analytical estimations of the self and mutual inductances require assumptions that restrict the use to machines with smooth air gap such as asynchronous machines or synchronous PM machines. It must also be remembered that the manner in which the flux density is valued is based on 2D hypothesis: so this method must be carefully used for machines with short axial length.

The major interest of the method presented in this paper is the possibility for the designer to predict the influence of the winding on crucial parameters (stator inductances) without considering dimensional parameters such as the stator radius, the stator or rotor yoke thickness... Concretely, from the knowledge of the phase, pole and slot numbers, the matrix modeling of the winding allows us to systematically and clearly predict the trend of the winding design on the set of inductance values. If the proposed approach is used for a wye-coupled three-phase machine, the interest is not great in this case because the machine can be considered as an equivalent two-phase machine characterized by a single synchronous inductance. For multiphase machines (we have chosen five phases in this paper), the approach allows us to quantify the ratio between the several synchronous inductances that characterize the machine. These ratio will have a direct impact on the choice of the carrier frequency of the PWM voltage source inverter that supplies the machine [16].

From a practical point of view, the matrix modeling is a really well-adapted support when performing algorithms to select winding distributions for given numbers of phases, poles and slots. Optimisation procedures can be developed regarding the winding factors, the inductance values and manufacturing constraints concerning end-windings notably $[16,17,19]$. Furthermore, the matrix winding modeling can also be used to study multi-star multi-phase windings [18]. From a theoretical point of view, the winding matrix modeling is a tool which can extend the multi-machine theory $[23,39]$ and thus to develop systematic design rules dedicated to multi-phase PM machines [40].

\section{Appendix A: Calculation of the total winding factor}

In this appendix, we aim to estimate the maximum value of the coil disposition factor $K_{d, h}$. It is well-known that this maximum value is obtained with a fully-pitched concentrated winding with $s_{p p}=1$ (as in Fig. 2 for example). First, we consider the case where the winding is a single coil with forward in slot numbered $f$ and backward in slot numbered $b$. The coil disposition factor is then:

$$
K_{d, h}=\left|2 j e^{-j \frac{2 \pi}{N_{s}} p h \frac{f+b}{2}} \sin \left(\frac{\pi}{N_{s}}(b-f) p h\right)\right| .
$$

More generally, if the winding is made up of $n_{c}$ coils characterized by their center position $\nu_{c}$ and their pitch $\tau_{c}$, the total coil disposition factor becomes:

$$
K_{d, h}=\left|\sum_{c=0}^{n_{c}-1} 2 j e^{-j \frac{2 \pi}{N_{s}} \nu_{c} p h} \sin \left(\frac{\pi}{N_{s}} \tau_{c} p h\right)\right| .
$$

For the classical case of the fully-pitched winding, the coil characteristics $\nu_{c}$ and $\tau_{c}$ can be expressed as follows:

$$
\left\{\begin{array}{l}
\nu_{c}=\frac{N_{s}}{2 p}+c \frac{N_{s}}{p} \\
\tau_{c}=\frac{N_{s}}{2 p}
\end{array}\right.
$$

As the total number of coils is equal to the pole pair number, the winding distribution factor can be expressed as follows:

$$
K_{d, h}=\left|\sum_{c=0}^{p-1} 2 j(-1)^{h} \sin \left(\frac{\pi}{2} h\right)\right|
$$


The traditional following conclusion can be drawn:

$$
\left\{\begin{array}{l}
h=2 k \quad \Longrightarrow K_{d, h}=0 \\
h=2 k+1 \Longrightarrow K_{d, h}=2 p=\frac{N_{s}}{N} .
\end{array}\right.
$$

By definition, for any winding, the total winding factor is the ratio between the coil disposition factor of the considered winding and the coil disposition factor of the equivalent fully-pitched configuration.

\section{Appendix B: First harmonic approach to estimate the set of inductances}

This classical method considers that the magnetomotive force is sinusoidal. In this case, the mutual air gap inductance $m_{n}^{a}$ can be deduced from the self air gap inductance $m_{0}^{a}$ :

$$
m_{n}^{a}=m_{0}^{a} \cos \left(\frac{2 \pi}{N} n\right)
$$

A leakage inductance is commonly added to the self air gap inductance but only for the self inductance:

$$
\left\{\begin{array}{l}
m_{0}=m_{0}^{a}+m_{0}^{l} \\
m_{n}=m_{0}^{a} \cos \left(\frac{2 \pi}{N} n\right) \forall n \in[1 \ldots N-1] .
\end{array}\right.
$$

The self air gap inductance can be estimated via relation (44):

$$
m_{0}^{a}=\frac{\mu_{0}}{g+g_{m}} L_{m} R_{s} n_{c d}^{2} \frac{2 \pi}{N_{s}} \sum_{m=0}^{N_{s}-1} w_{m, 0}^{2} .
$$

The leakage inductance $m_{0}^{l}$ can be estimated from relation (48):

$$
m_{0}^{l}=\mu_{0} n_{c d}^{2}\left(\frac{p_{s}}{3\left(R_{s}+b_{s}+p_{s}\right) \delta_{s}}+\frac{b_{s}}{R_{s} \sigma_{s}}\right) \sum_{m=0}^{N_{s}-1} d_{m, 0}^{2}
$$

The approach described in this paper differs from the classical one for two reasons. On the one hand, concerning inside air gap flux density couplings between phases, all the magnetomotive force harmonics are taken into account. On the other hand, concerning outside air gap flux density couplings between phases, a modeling is introduced.

\section{Appendix C: Self-inductance and mutual inductance measurements}

For both machines, an impedancemeter (WaybeKerr type) and a $15 \mathrm{~W} 100 \mathrm{~Hz}$-frequency generator are used to measure the winding self-inductance and the set of mutual inductances. Each phase is supplied to estimate the selfinductances and the mutual inductances. In order to evaluate uncertainty in measurement, the operation is repeated for numerous positions of the rotor (10 positions
Table 7. Inductance results measurements obtained for the M5-A machine.

\begin{tabular}{c|ccc}
\hline Inductance & $m_{0}$ & $m_{1}$ & $m_{2}$ \\
\hline Measurement $(\mathrm{mH})$ & 1.31 & 0.20 & -0.52 \\
Uncertainty & $5 \%$ & $29 \%$ & $17 \%$ \\
\hline
\end{tabular}

Table 8. Inductance results measurements obtained for the M5-B machine.

\begin{tabular}{c|ccc}
\hline Inductance & $m_{0}$ & $m_{1}$ & $m_{2}$ \\
\hline Measurement $(\mathrm{mH})$ & 1.13 & 0.03 & -0.17 \\
Uncertainty & $10 \%$ & $9 \%$ & $4 \%$ \\
\hline
\end{tabular}

precisely). Tables 7 and 8 give the results obtained for M5-A and M5-B machines including uncertainty in the measurement. For each inductance, the measurement uncertainty is considered to be the standard deviation of the whole measurements.

For the M5-A machine, the rather important measurement uncertainty regarding the two mutual inductances comes from a stronger than expected saliency effect.

\section{References}

1. J. Hendershot, Design of brushless permanent magnet motors (Clarendon Press Oxford, 1994), Chap. 3

2. D. White, H. Woodson, Electromechanical Energy Conversion (John Wiley \& Sons, 1959), Chap. X

3. N. Bianchi, M.D. Pré, Proc. IEE Electr. Power Appl. 153, 459 (2006)

4. J. Cros, P. Viarouge, IEEE Trans. Energy Conv. 17, 248 (2002)

5. A.M. El-Refaie, T.M. Jahns, D.W. Novotny, IEEE Trans. Energy Conv. 21, 34 (2006)

6. F. Libert, J. Soulard, Investigation on Pole-Slot Combinations for Permanent-Magnet Machines with Concentrated Windings, in 16th Int. Conf. on Electrical Machines, ICEM 2004, Cracow, Poland

7. F. Magnussen, C. Sadarangani, Winding factors and Joule losses of permanent magnet machines with concentrated windings, in Electric Machines and Drives Conf. IEMDC (IEEE, 2003), Vol. 21, pp. 333-339

8. N. Bianchi, S. Bolognani, E. Fornasierao, A general approach to determine the rotor losses in Three-phase fractional-slot PM machines, in Electric Machines and Drives Conf. IEMDC (IEEE, 2007), Vol. 1, pp. 634-641

9. F. Magnussen, H. Lendenmann, Parasitic effects inPM machines with concentrated windings, in IAS Annual Meeting (2005), Vol. 2, pp. 1044-1049

10. Y.S. Chen, Z.Q. Zhu, D. Howe, IEEE Trans. Magn. 42, 3395 (2006)

11. L. Parsa, On Advantage of Multi-Phase Machines, in 31st Annual Conf. of IEEE Industrial Electronics Society, IEEE-IECON, 2005, p. 6

12. E. Levi, E. Bojoi, F. Profumo, H. Toliyat, S. Williamson, IET Electr. Power Appl. 1, 489 (2007)

13. E. Levi, IEEE Trans. Ind. Electron. 55, 1893 (2008)

14. J. Figueroa, J. Cros, P. Viarouge, IEEE Trans. Energy Conv. 21, 1 (2006) 
15. H.M. Ryu, J.H. Kim, S.K. Sul, Synchronous Frame Current Control of Multi-Phase Synchronous Motor, Part I, Modeling and current control based on multiple d-q spaces concept under balanced condition in IEEE 39th IAS Annual Meeting 2004, Seattle, USA, Vol.1, p. 63

16. F. Scuiller, E. Semail, J.F. Charpentier, P. Letellier, IET Electr. Power Appl. 3, 102 (2009)

17. F. Scuiller, J.F. Charpentier, E. Semail, S. Clénet, A multiphase surface mounted permanent magnet design to reduce Torque Ripples and Joule losses for naval applications, in Proc. of the All Electric Ship Symposium, Versailles, France, 2005

18. F. Scuiller, J.F. Charpentier, E. Semail, S. Clénet, P. Letellier, Study of unconventional winding configuration of multiphase permanent magnet synchronous machine to improve reliability and torque quality for pod propulsion application, in Proc. 2nd Int. Conf. on Technological in Podded Propulsion, TPOD2006, Brest, France, 2006

19. F. Scuiller, J. Charpentier, E. Semail, S. Clénet, A global design strategy for multiphase machine applied to the design of a 7-phase fractional slot concentrated winding PM machine, in Proc. of ICEM'06 Conf., Chania, Greece, 2006

20. H.A. Toliyat, T.A. Lipo, J.C. White, IEEE Trans. Energy Conv. 6, 679 (1991)

21. H.A. Toliyat, T.A. Lipo, J.C. White, IEEE Trans. Energy Conv. 6, 684 (1991)

22. B. Mecrow, A. Jack, J. Haylock, J. Coles, Proc. IEE Electr. Power Appl. 143, 437 (1996)

23. E. Semail, A. Bouscayrol, J.P. Hautier, Eur. Phys. J. Appl. Phys. 22, 207 (2003)

24. L. Parsa, H.A. Toliyat, Five-Phase Interior Permanent Motor with Low Torque Pulsation, in Proc. of IEEEIASó5, Hong-Kong, 2005, Vol. 3, pp. 1770-1775

25. E. Semail, X. Kestelyn, A. Bouscayrol, Right Harmonic Spectrum for the Back-Electromotive Force of a $N$-phase Synchronous Motor, in IEEE-IAS'04, Seattle, USA, 2004. Vol. 1, pp. $71-78$
26. J.P. Martin, F. Meibody-Tabar, B. Davat, Multiplephase Permanent Magnet Synchronous Machine supplied by VSIs working under fault conditions, in Industry Applications Conf. (IEEE, 2000), Vol. 3, pp. 1710-1717

27. F. Locment, E. Semail, F. Piriou, IEEE Trans. Magn. 42 1427 (2006)

28. E. Klingshirn, IEEE Trans. Power App. Syst. PAS-102, $47(1983)$

29. X. Kestelyn, E. Semail, J.P. Hautier, Vectorial multimachine modeling for a five-phase machine, in Int. Conf. on Electrical Machine ICEM, Belgium, 2002

30. J. Pyrhonen, T. Jokinen, V. Hrabovcova, Design of rotating electrical machines, 1st edn. (Wiley, 2008)

31. J. Saint-Michel, Technique de l'Ingénieur D3420, 1 (2001)

32. Z.Q. Zhu, D. Howe, IEEE Trans. Energy Conv. 15, 407 (2000)

33. Z.Q. Zhu, D. Howe, IEEE Trans. Magn. 29, 136 (1993)

34. M. Bekemans, Ph.D. thesis, Université catholique de Louvain, 2006

35. M. Bekemans, F. Labrique, E. Matagne, Accurate Torque Control of VSI Fed Synchronous Machine Under Normal or Fault Conditions Using Discrete Modelling of Airgap Flux, in Proc. ICEM'06 Conf., Chania, Greece, 2006, pp. 344-1-344-6

36. Z.Q. Zhu, D. Howe, IEEE Trans. Magn. 29, 143 (1993)

37. J.L. Kirtley, Class Notes 5, Massachussets Institute of Technology, Department of Electrical Engineering and Computer Science, 1997

38. A. Foggia, Techniques de l'Ingénieur D3440, 1 (1999)

39. E. Semail, Ph.D. thesis, Université des Sciences et des Technologies de Lille, France, 2000

40. F. Scuiller, Ph.D. thesis, École Doctorale de l'École Nationale Supérieure d'Arts et Métiers, 2006 